LAWRENCE LIVERMORE NAT IO N A L LABORATORY

\section{Understanding of Edge Plasmas in Magnetic Fusion Energy Devices}

T.D. Rognlien

November 2, 2004

Plasma Physics and Controlled Fusion 
This document was prepared as an account of work sponsored by an agency of the United States Government. Neither the United States Government nor the University of California nor any of their employees, makes any warranty, express or implied, or assumes any legal liability or responsibility for the accuracy, completeness, or usefulness of any information, apparatus, product, or process disclosed, or represents that its use would not infringe privately owned rights. Reference herein to any specific commercial product, process, or service by trade name, trademark, manufacturer, or otherwise, does not necessarily constitute or imply its endorsement, recommendation, or favoring by the United States Government or the University of California. The views and opinions of authors expressed herein do not necessarily state or reflect those of the United States Government or the University of California, and shall not be used for advertising or product endorsement purposes. 


\title{
Understanding of edge plasmas in magnetic fusion energy devices
}

\section{T.D. Rognlien}

University of California Lawrence Livermore National Laboratory, Livermore, California 94551 USA

\begin{abstract}
A limited overview is given of the theoretical understanding of edge plasmas in fusion devices. This plasma occupies the thin region between the hot core plasma and material walls in magnetically confinement configurations. The region is often formed by a change in magnetic topology from close magnetic field lines (i.e., the core region) and open field lines that contact material surfaces (i.e., the scrape-off layer $[\mathrm{SOL}]$ ), with the most common example being magnetically diverted tokamaks. The physics of this region is determined by the interaction of plasma with neutral gas in the presence of plasma turbulence, with impurity radiation being an important component. Recent advances in modeling strong, intermittent micro-turbulent edgeplasma transport is given, and the closely coupled self-consistent evolution of the edge-plasma profiles in tokamaks. In addition, selected new results are given for the characterization of edge-plasmas behavior in the areas of edge-pedestal relaxation and SOL transport via Edge-Localize Modes (ELMs), impurity formation including dust, and magnetic field-line stochasticity in tokamaks.
\end{abstract}

PACS numbers: $52.40 . \mathrm{H}, 52.55 . \mathrm{Dy}, 52.55 . \mathrm{Fa}, 52.75 .-\mathrm{d}$ 


\section{Introduction and motivation}

The distribution of plasma fluxes to material surfaces is a key issue for fusion devices because it identifies peak heat loads, and determines hydrogenic and impurity particle sources from recycling and sputtering. The wall fluxes can erode the material, setting its lifetime, and release potentially undesirable impurities into the plasma discharge. In a fusion reactor, helium ash must be removed, and tritium must be recoverable in a straightforward manner. In addition to these "standard" edge-plasma issues, there is now extensive experimental and theoretical evidence that edge-plasma parameters can have a profound effect on the core energy confinement, which further elevates the need to understand the physics of the edge region in detail. A thorough discussion of edge plasma issues relative to the planned ITER device as of the late 1990's can be found in Ref. [1].

Here progress on a selected set of edge-plasma topics is discussed with a limited number of references. Thus, the paper only presents a short glimpse of recent developments and is not meant to be represented as a thorough review. The plan of the paper is as follows: In Sec. 2, the geometry, scales and equations for the edge are discussed. Section 3 traces the development of radial electric field calculations and transport simulations. Microinstabilities and turbulence are considered in Sec. 4, while longer wavelength magnetohydrodynamic (MHD) modes are discussed in Sec. 5. Developments in sheath and wall physics, as well as configurational innovations, are given in Secs. 6 and 7. The paper concludes with a short summary in Sec. 8.

\section{Geometry, spatio-temporal scales, and basic equations}

Here we focus our attention on tokamaks because this configuration has been the subject of most experimental and theoretical research in the last 40 years. In terms of complexity, tokamaks [2] also have the virtue of being nearly toroidally symmetric. In addition, many of the basic features of tokamak edge-plasmas have a close parallel in stellarators[3] that are experiencing a resurgence [e.g., W7-AS, LHD, NCSX, and W7-X].

Edge plasmas encompass both a closed magnetic-field (B-field) line region adjacent to the a material limiter in "limiter" tokamaks, or the magnetic separatrix in divertor tokamaks as show in 1 for the DIII-D tokamak. The coordinate forming the nested surfaces shown in Fig. 1. in the core region are poloidal magnetic flux surfaces in which the total magnetic field lies. These surfaces are the output of the 2D MHD equilibrium code EFIT [4]. Outside the limiter or separatrix, the field lines pass through material surfaces, which results in important plasma sheath and plasma/wall interactions, including high heat fluxes.

The change from closed to open B-field lines results in radial plasma gradients at

the edge typically being very steep owing to the abrupt introduction of rapid plasma loss on the open B-field lines. A sketch of a typical radial profile and identification of 
two regions, the pedestal and scrape-off layer (SOL) are shown in Fig. 2. The steepness of gradients in the edge region can drive various strong instabilities, but can also give rise to rotational shear the help reduce the turbulence fluctuation level and resulting transport. Such action of turbulence suppression produces what is know as a transport barrier that is of key importance for improving confinement in tokamaks as discussed later. The rapid change in plasma variables in the edge also extends the range of possible spatial and temporal scales that must be consider for theoretical models. An example of these is shown in Fig. 3 corresponding to the outer midplane region of the DIII-D tokamak. In the close-field region, plasma parameter tend to be "flux functions" meaning that they are nearly constant on a magnetic flux surface. However, in the SOL, strong variations along the B-field can occur, especially near the divertor or limiter plates where lower temperatures and higher densities (preserving parallel pressure balance) at typical. Generally, three regimes are identified, known as detached, high-recycling, and low-recycling or sheath-limited, are discussed elsewhere, e.g., Ref. [5] and [6].

The basic equation to describe the edge-region plasma formally begins with the 6D (3 each in configuration and velocity spaces) equation for the electron and ion distribution functions including Coulomb collisions. Owing to the complexity of this equation, most work has been done with reduced moment descriptions, a common example be Braginskii [7], which yield 3D moment or fluid equations for density, momentum, and temperature. Such a procedure requires localization in the parallel and cross-field directions to obtain a closure relation for the higher moments. Thus, the radial gradients must be much smaller than the gyro radius and the collisional meanfree path must be short along B for validity. Within the last five years, interest and use of a similar set of equations [8] has arisen that differs in that the ordering used to reduce the kinetic equation is that the flow velocity is assumed small compared to the ion thermal velocity, whereas Braginskii [7] assumes the flow velocity is comparable to the thermal speed. One of the main differences that the parallel ion viscosity then include as contribution from the ion heat flux. However, very recently, Simakov and Catto [9] have corrected some errors in Ref. [8] to obtain an even more rigorous set of fluid equations.

Work for a more complex reduced kinetic equation for the edge has not yet advanced to the same level of completeness as the fluid equations for the short mean-free path regimes. However, many existing tokamaks are already in the long mean-free path regime, especially in the pedestal region, and this will be even more true for future devices such as ITER. For tokamak core plasmas, the gyrokinetic equation performs an appropriate average of the gyrophase to yield a 5D equation (e.g., [10]). However, the core plasma ordering is typically for small radial gradients and weak fluctuations, while in the edge, this ordering must be relaxed. Some progress in this extension is beginning to be reported [11]. 


\section{Calculations of electric fields and plasma profiles}

Fluid equations have been used by numerous groups in the last 20 years to determine plasma profiles in the edge region, usually with the ad-hoc inclusion of turbulent radial transport; a topic discussed in Sec. 4. Here we discuss the evolution of models for the radial electric field extending from the SOL to inside the closed field-line region. For a 2D slab model, it was shown that by including the perpendicular ion viscosity, one could obtain a connection between the radial electric field in the closed and open field-line regions [12]. Here the classical Braginskii collisional radial transport of density, momentum, and energy were all consistently enhanced by the same factor. Subsequently, this model was implemented for toroidal geometry, including the important effect of $\nabla B$ and curvature currents [13]. These models are full transport simulations giving profiles of density, parallel momentum, and electron and ion temperatures. Simulations show that the only very near the separatrix is $E_{r}$ sensitive an order of magnitude change in the viscosity coefficient, whereas changing the particle and energy diffusivities signicantly changes the plasma profiles and consequently $E_{r}$ owing to the need to satisfy the perpendicular ion momentum equation [14].

Then this type of analysis was extend by Rozhansky et al. [15] to include the Mikhailovskii set of equations, where the appearance of the heat flux in the viscosity also allowed the resolution of the electric field cross the separatrix. This work did not include an anomalous turbulent viscosity, even though anomalous radial particle and heat diffusivities were used for the transport simulations. The resulting $E_{r}$ profiles are qualitatively similar to the early work, but a detailed comparison has not appeared. These authors also pointed out that the resulting $E_{r}$ in the pedestal was similar to the analytic neoclassical collisional model of Hirshman and Sigmar [16] that contains no turbulence-enhanced transport. However, this result is not so quantitatively different from the ion perpendicular moment balance that is built into the simulation, so the result is not unexpected.

Turning back to analytic theory, very recently, Simakov and Catto [17] have used their improved fluid theory the corrected previous omissions to obtain a new, analytic description of the neoclassical $E_{r}$ in this collisional regime. Quantitative comparison with previous models of $E_{r}$ profiles for transport calculations of specific cases is yet to be done.

To understand the impact of the less collisional regime of edge operation when ions (and electrons) can complete their poloidal drift orbits (banana orbits) before a significant collisional deflection, some researchers have utilized Monte Carlo ion simulations $[18,19]$ that include guiding-center orbits and Coulomb collisions. For these simulations, the radial electric field inside the separatrix is determined by combining Poisson's equation and current continuity to yield (in SI units)

$$
\frac{\partial E_{r}}{\partial t}=-j_{r} / \epsilon
$$

where $j_{r}$ is the radial current deduced from the simulations and $\epsilon$ is the plasma 
permitivity. This equation is flux-surfaced averaged to give a flux-surface average of $E_{r}$. For the SOL, ad hoc models are used for $E_{r}$, although here the determination of $E_{r}$ should be largely controlled by the parallel electron physics that could be added to such simulations.

The goal of these simulations is to determine neoclassical loss rates in the edge where analytic theory is difficult, and in particular, the impact of ion orbit loss. In fact, such orbit loss and its effect on $E_{r}$ has been proposed [20] as a mechanism for the L-H transition discussed in the next section. The results do typically show a steep, negative $E_{r}$ in the pedestal region as do the transport models shown above. Specific comparisons with the standard neoclassical loss rates [16], which while not srictly valid for the steep edge region, would help quantify the differences.

\section{Micro-turbulence - drift instabilities and blobs}

Many possible modes of low-frequency microinstabilities have been identified in the edge plasma owing to steep gradients, flow flows, and sheath resistance (e.g., Ref. [21]). Because density gradients tend to be at least as strong as ion temperature gradients, one of the dominant instabilities appears to be resistive the drift-wave branch driven unstable by resistivity, rather than the ion temperature gradient that is often believed to be a important component of core microinstabilities.

Because of the complexity of plasma turbulence, detailed studies tend to be carried out with 3D fluid simulation codes. Some of these in present use are the BOUT code [22], which includes both the pedestal, x-point, and SOL plasmas for tokamak geometry with electromagentic corrections, the DRBM code [23], which follows a flux-tube within the pedestal with electromagnetic corrections, and the DALF code [24], which also follows a flux-tube within the pedestal. Aspects of such turbulence have also been studied extensively in 2D codes, e.g. Ref. [25].

For tokamak geometries, it has been shown [26, 27] that magnetic shear near the xpoint regions can have a strong impact on the drift modes. In particular, a large increase in the perpendicular wavenumber of the eigenfuction occurs from flux-tube distortion, and this in turn, increases the effective resistivity of the plasma in the vicinity of the $\mathrm{x}$-point. There is a combined effect of increasing the growth rate of the resistive drift wave and in forcing the eigenfunction to small values at the x-point. The effect on the resistivity and eigenfuction are shown in Fig. 4 for a single-null divertor configuration.

A feature of SOL turbulence at high turbulence level (which typically increases with plasma density) is the occurrence of rapid outward convection of plasma. Such behavior has been observed or inferred by various diagnostics, such as Langmuir probes, Gas-Puff imaging, and imaging of background $\mathrm{H}_{\alpha}$ light ( $[28,29]$ and references therein). Analysis of polarization of plasma density "blobs" from opposite ion and electron $\nabla B$

drifts, and the resulting $\mathbf{E} \times \mathbf{B}$ drift, provides a simple explanation of the rapid outward motion [30]. However, understanding the growth and saturation of "blobs," as well as more complex dynamics, requires full turbulence simulations. The impact of such strong 
radial transport has been investigated in transport simulations by postulating a rapidly increase outward convection of hydrogen plasma in the SOL and by a mix of inward convection (low charge state) and outward convection (high charge state) for a carbon impurity [31].

The strong turbulence in the edge plasma has a large effect on the plasma profiles, whose gradients drive the underlying instabilities. Thus, to obtain a self-consistent description of edge plasma profiles, it is important to couple the turbulence simulations with plasma transport. Progress in this area has been made recently by evolving profiles directly as a component of the turbulence simulations, both in $2 \mathrm{D}[25]$ and in $3 \mathrm{D}$ [32]. Such simulations are limited to the timescale that it is possible to run the full time simulations on present-day computers. Because profile evolution in the SOL can have very long timescales owing to particle recycling and impurity production from walls, an approach to extend the coupling has been implement that uses time-averaged turbulence fluxes in a 2D transport simulation, and then updates the profiles used for the 3D turbulence simulation in an iterative fashion until a steady-state is obtained. An example of the latter process for the density profile at the outer midplane is shown in Fig. 5 using the BOUT turbulence and UEDGE transport codes [33]. For this particular case, the strong convective transport can effectively described by a radially increased diffusion coefficient; more generally, a combination of convection and diffusion is used. As long as the transport code faithfully represents the turbulent plasma flux from the $3 \mathrm{D}$ code, the split into convection and diffusion is a matter of numerical stability and convenience.

An important aspect of edge turbulence and the resulting enhanced transport is its strong suppression in the pedestal region during what is termed the high-confinement, or H-mode operation. The reduction of turbulent transport is know as a transport barrier. There are many theories proposed to explain this L-H mode transition as discussed in Ref. [35]. However, there is yet to be a definitive identification of the cause, and indeed, there may be different mechanism dominating for different regimes. Many believe that the shear in the radial electric field in the pedestal region, discussed in Sec. 3 is an important component. Owing to the complex nature of the edge, it seems likely that a combination of turbulence/transport simulations, detailed analysis, and close comparison with experiments will be required to fully understand the L-H transition.

\section{Macro-turbulence - Edge-Localized Modes}

A common feature of tokamak operation is the H-mode just described where the microturbulence in the pedestal region is substantially reduced. This allows the plasma temperature and density in the pedestal region to increase substantially. However, because the parallel loss on open B-field lines is still operable, the density and energy can quickly flow to the divertor or limiter there. The net result is that the H-mode is characterized by steeper plasma profiles than in the more turbulent L-mode. In many discharges, it appears that the ultimate edge gradients are limited by the onset of Edge- 
Localized Modes (ELMs) that cause periodic (few to hundreds of millisecs), large-scale edge relaxation events where the edge plasma is injected into the SOL. A summary of some of the experimental measurements of ELM properties is given in Ref, [34].

There has been substantial progress made in the last 5 years to identify ELMs as low toroidal mode number (say, $n_{t} \leq 40$ ) MHD instabilities driven by pressure gradients (ballooning modes) and strong bootstrap edge currents (peeling modes), themselves driven by radial gradients. Comparison with experiments has been greatly aided by linear stability codes, especially ELITE [36, 37] and MISKA [38] that include the 2D MHD equilibrium and flux-surface-averaged plasma profiles up to the separatrix. An example of a typical ELM plasma-displacement eigenfunction from ELITE for DIII$\mathrm{D}$ that emphasizes the ballooning character is shown in Fig. 6a from Ref. [39]; note the ballooning structure where the eigenfunction is largest on the outside of the torus where magnetic curvature is destabilizing. Correspondences between ELM threshold versus a number of plasma parameters have been obtained, which present a compelling argument that such MHD modes are indeed the source of ELMs. For example, the comparison between the ELITE stability boundary and plasma density is shown in Fig. 6b Similar correspondences are obtained versus plasma current and triangularity of the MHD equilibrium; some other studies have found a similar correspondence for devices such as JT-60U.

The nonlinear saturation and transport cause by the ELM is much less well understood. There is some analytical study of weakly nonlinear ELMs that shows that they may first become even more unstable owing to nonlinear processes before they ultimately saturate [40]. Some 3D nonlinear fluid simulations have also been done into the weakly nonlinear phase (before the code develops numerical problems) [41] that suggest that fast radial transport may take place, but this area needs considerable more development. replenishment The transport of energy and particles to the divertor from ELM events is of great practical interest for future devices such as ITER. Owing to the high plasma temperatures, kinetic effects can play a significant role. This is the topic of another paper at this conference [42].

\section{Sheath and wall effects}

Plasma sheaths have received considerable attention over the years, even with the complication of the small angle that the field line typically makes with the material surface in tokamaks. As long as the angle of the field exceeds roughly the square root of the electron and ion mass ratio, a "standard" sheath develops consisting of two regions: the electrostatic sheath adjacent to the surface on the order of the electron Debye length, and the larger ion gyro radius sheath corresponding to gyrating ions intersection the surface in their cyclotron orbit.

A potentially important effect that has been analyzed in some detail is what happens with rough, random surfaces that can arise owing to erosion and redeposition on the surface [43]. For roughness between the Debye length and the ion gyroradius, 
substantial changes can take place in the sheath structure. These effects have not yet been incorporated into standard edge modeling simulations.

Another area of interest and concern is the production and transport of dust particles by nucleation and chemical processes in the volume or on the surface [44]. These particles can present a large source of impurities, and topic is in more detail in another paper at this conference [45].

\section{Innovations}

Lastly, it is important to at least note that there have been a number of new ideas emerge to manipulate the edge plasma region in a way that minimized the negative influence of high heat fluxes, material erosion, and plasma stability and performance; here we mention a few.

The use of liquid walls/divertors has received some study, both as a means of reducing the erosion problem by continuous replenishment and as a way of improving plasma performance [46] if substantial particle pumping can occur, as for lithium. For molten salts such as flibe (a mixuture of fluorine, lithium, and beryllium), thickness on the order of $1 \mathrm{~m}$ can also attentuate much of the neutral flux, thus reducing structural material activation. For the liquids, an important issue is the impurity contamination of the core plasma from the liquid vapor. Transport simulations indicated that the fluorine from flibe can be a problem unless operating at high plasma density (above $10^{20} \mathrm{~m}^{-} 3$ ) [47]. Lithium may be tolerable in present devices, but more analysis is needed that includes the transport in the low-recycling edge plasma so produced [48]

Another idea to reduce heat loads is to produce a second X-point some distance farther away from the main plasma than the first X-point, but adjacent to it [49]. Here impurities could be injected to radiate much of the exhaust power, but it is argued that such a radiating region will be spatially stable, unlike the convention single X-point configuration where strong impurity radiation can lead to a radiation instability for the core known as a MARFE.

A scheme for enhancing SOL-only transport in order to spread the heat flux on

the divertor plate utilizes introduction of toroidally asymmetric spatial biasing or nonuniformities in the divertor to produce essential convective cells in the SOL [50]. This is now being used in the MAST experiment at Culham with understandable results [51].

Finally, we mention work to modify ELMs by non asymmetric magnetic coil perturbations. This work has been carried out at DIII-D by Evans and co-workers [52]. For modest coil currents, the frequency and amplitude of ELMs can be substantially modified. The mechanisms under consideration are keeping the edge plasma gradients below the critical value for ELMs by inducing enhance spatial plasma transport through stochastic 3D fields, and the other is direct interaction between the the imposed 3D fields and the MHD modes to reduce the instability drive. 


\section{Summary}

A brief overview of advances in a selected set of edge-plasma topics has been given. The paper has focused on developments in theory and computation. Topics covered range from basic plasma equations, transport simulations and calculation of the radial electric field, micro- and macro-turbulence, sheath effects, and innovations. There have also been important advances in atomic/molecular physics and radiation transport that space limitations did not allow to be covered here. Why a great deal of progress has been made, it seems clear that much more theoretical analysis and simulation development is needed before on can predict and perhaps control the behavior of edge plasmas in future devices.

\section{Acknowledgments}

We thank R.H. Cohen, S.I. Krasheninnikov, and D.D. Ryutov for helpful discussions. This work was performed under the auspices of the U.S. Dept. of Energy by the University of California Lawrence Livermore National Laboratory under contract No. W7405-Eng-48 and was supported by LDRD project 03-ERD-009.

\section{References}

[1] ITER Physics Basis, in Nucl. Fusion 39 (1999), Chapt. 4, 2391.

[2] J. Wesson, Tokamaks 2nd ed. Oxford: Claredon Press; New York: Oxford University Press, 1997.

[3] G. Grieger, W. Lotz, P. Merkel, et al., Phys. Fluids B 4 (1992) 2081.

[4] L.L. Lao, J.R. Ferron, R.J. Groebner, et al., Nucl. Fusion 30 (1990) 1035.

[5] Peter C. Stangeby, The Plasma Boundary of Magnetic Fusion Devices (Inst. Physics Pub., Bristol and Philadelphia, 2000).

[6] T.D. Rognlien and M.E. Rensink, Fusion Eng. Design 60 (2002) 497.

[7] S.I. Braginskii, Transport processes in a plasma, Reviews of Plasma Physics, Vol. 1, Ed. M.A. Leontovich (Consultants Bureau, New York, 1965), p. 205.

[8] A.B. Mikhailovskii and V.S. Tsypin, Beitr. Plasmaphys. 24 (1984) 335.

[9] A.N. Simakov and P.J. Catto, Phys. Plasmas, to be pub., May 2004.

[10] T.S. Hahm, Phys. Plasmas 3 (1996) 4368.

[11] W.M. Nevins, T.S. Hahm, H. Qin et al., Bull. Am. Phys. Soc. 2004.

[12] T.D. Rognlien and D.D. Ryutov, Plasma Phys. Repts. 25 (1999) 1024.

[13] T.D. Rognlien, D.D. Ryutov, N. Mattor, and G.D. Porter, Phys. Plasmas 6 (1999) 1851.

[14] T.D. Rognlien, X.Q. Xu, and R.H. Cohen, Plasma Phys. Contr. Fusion 42 (2000) A271.

[15] V. Rozhansky, et al. , Nucl. Fusion 412001387.

[16] S.P. Hirschman and D.J. Sigmar, Nucl. Fusion 21 (1981) 1079.

[17] A.N. Simakov and P.J. Catto, Phys. Plasmas, to be pub. (2004).

[18] J.A. Heikkinen, T.P. Kiviniemi, T. Kurki-Suonio, et. al. , J. Comp. Phys. 173 (2001) 527.

[19] C.S. Chang, Seunghoe Ku, and H. Weitzner, Phys. Plasmas. 11 (2004) 2649.

[20] K.C. Shaing and E.C. Crume, Jr., Phys. Rev. Lett. 63 (1989) 2369.

[21] R.H. Cohen, N. Mattor, and X.Q. Xu, Contr. Plasma Phys. 34 (1994) 232.

[22] X.Q. Xu, R.H. Cohen, T.D. Rognlien, and J.R. Myra, Phys. Plasmas 7 (2000) 1951.

[23] B.N. Rogers, J.F. Drake and A. Zeiler Phys. Rev. Lett. 81 (1998) 4396.

[24] B. Scott, Phys. Fluids B 4 (1992) 2468. 
[25] Y. Sarazin, Ph. Ghendrih, G. Attuel et al., J. Nucl. Mater. 313-316 (2003) 796.

[26] D. Farina, R. Pozzoli, and D.D. Ryutov, Nucl. Fusion 33 (1993) 1317.

[27] J.R. Myra, D.A. D'Ippolito, X.Q. Xu, and R.H. Cohen, Phys. Plasmas 7 (2000) 2290.

[28] B. LaBombard, M.V. Umansky, R.L. Boivin et al., Nucl. Fusion 40 (2000) 2041.

[29] J.A. Boedo, D.L. Rudakov, R.A. Moyer et al., Phys. Plasmas 10 (2003) 1670.

[30] S.I. Krasheninnikov, Phys. Lett. A 283 (2001) 368.

[31] A.Yu. Pigarov, S.I. Krasheninnikov, T.D. Rognlien et al., Contrib. Plasma Phys. 44 (2004) 228.

[32] X.Q. Xu, W.M. Nevins, R.H. Cohen et al., Contrib. Plasma Phys. 44 (2004) 105.

[33] T.D. Rognlien, M.V. Umansky, X.Q. Xu, and R.H. Cohen, Contrib. Plasma Phys. 44 (2004) 188; also, J. Nucl. Mater., to be pub. (2004).

[34] A. Loarte, G, Saibene, R. Sartoni et al., Phys. Plasmas 11 (2004) 2668.

[35] J. W. Connor and H.R. Wilson, Plasma Phys. Control. Fusion 42 (2000) R1.

[36] H.R. Wilson, J.W. Connor, A.R. Field et al., Phys. Plasmas 6 (1999) 1925.

[37] P.B. Snyder, H.R. Wilson, J.R. Ferron et al., Phys. Plasmas 9 (2002) 2037.

[38] G.T.A. Huysmans et al., Plasma Phys. 8 (2002) 4292.

[39] P.B. Snyder, H.R. Wilson, T.H. Osborne, and A.W. Leonard, Plasma Phys. Control. Fusion 46 (2004) A131.

[40] H.R. Wilson and S.C. Cowley, Phys. Rev. Lett. 92 (2004) 175006-1.

[41] P.B. Snyder, H.R. Wilson, X.Q. Xu, and A.J. Webster, 31th EPS Conf. Plasma Phys., London, 28 June - 2 July, ECA 28G (2004) 2.156.

[42] D. Tskhakaya, this ICPP conference.

[43] R.H. Cohen and D.D. Ryutov, Contrib. Plasma Phys. 40 (2000) 456.

[44] S.I. Krasheninnikov, Y, Tomita, R.D. Smirnov, and R.K. Janev, Phys. Plasmas 11 (2004) 3141.

[45] S.I. Krasheninnikov, this ICPP conference.

[46] S.I. Krasheninnikov, L.E. Zakharov, and G.V. Pereverzev, Phys. Plasmas 43 (2003) 1678.

[47] T.D. Rognlien and M.E. Rensink, J. Nucl. Mater. 209-293 (2001) 312.

[48] J.N. Brooks, J.P. Allain et al., J. Nucl. Mater., to be pub. (2004).

[49] M. Kotschenreuther et al., IAEA Fusion Energy Conf., Vilamoura, Portugal (2004).

[50] R.H. Cohen and D.D. Ryutov, Nucl. Fusion 37 (1997) 621.

[51] S. Fielding et al., MAST paper (2003).

[52] T.E. Evans et al., Phys. Rev. Lett. 92 (2004) 235003-1. 


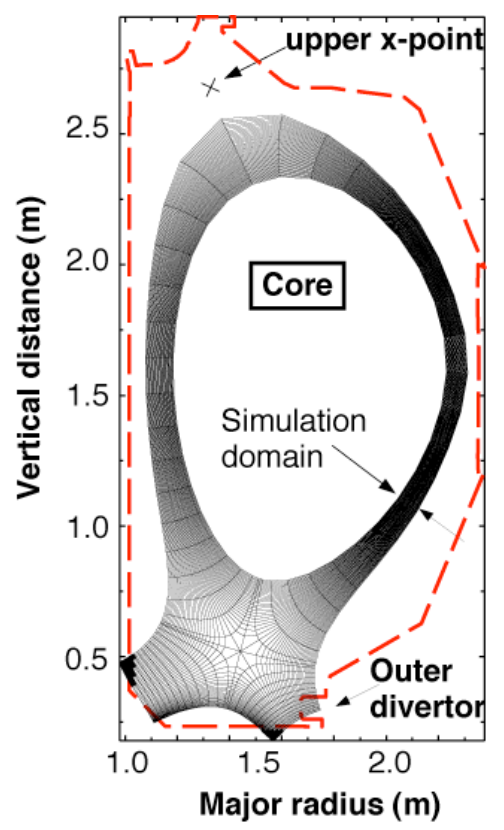

Figure 1. The edge-plasma domain for a typical DIII-D single-null discharge; the nearly concentric surfaces in the core region correspond to poloidal magnet flux surfaces. The chamber wall is shown in red. 


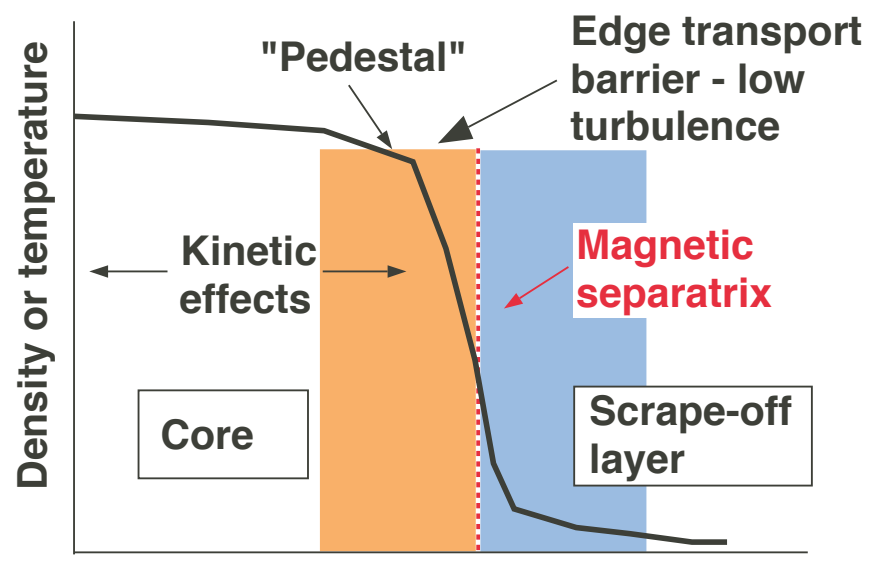

Radial position

Figure 2. The edge region at the outer midplane showing the pedestal, separatrix, and scrape-off layer. 

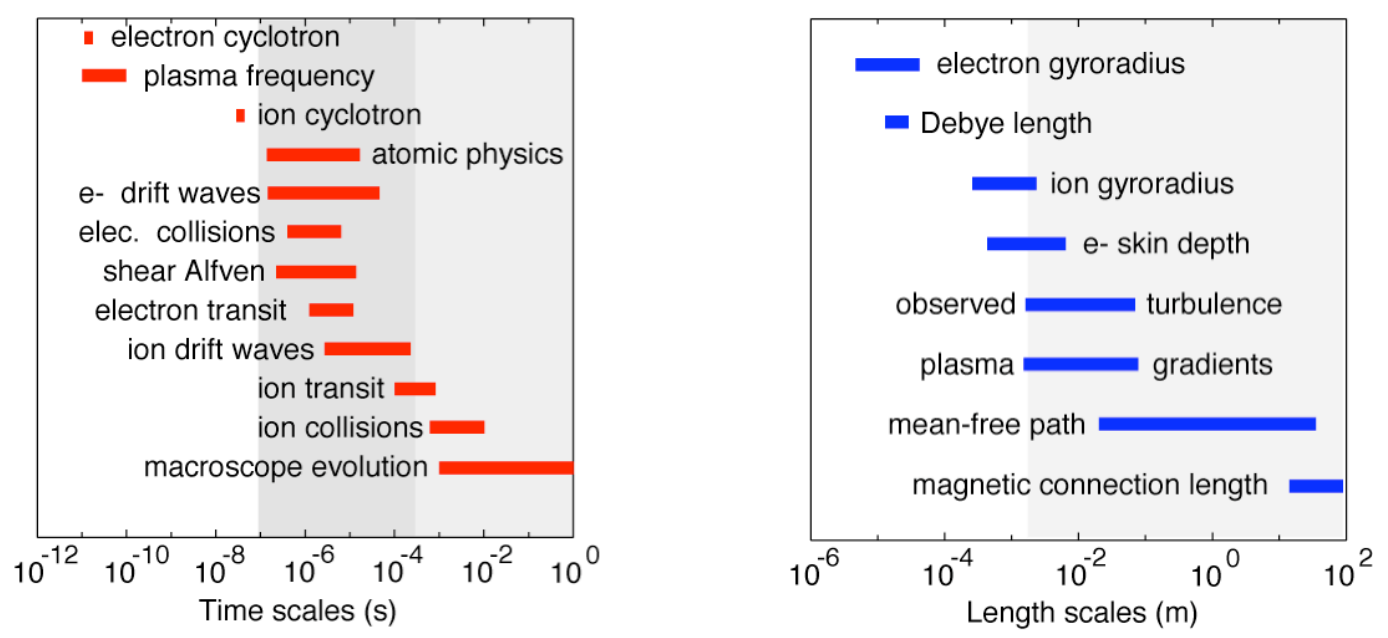

Figure 3. Time and spatial scales for edge plasmas (courtesy, P.B. Snyder, 2004)

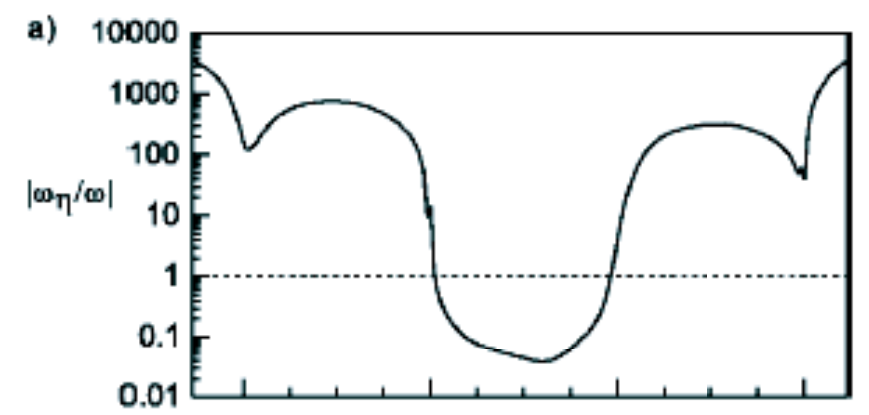

b)

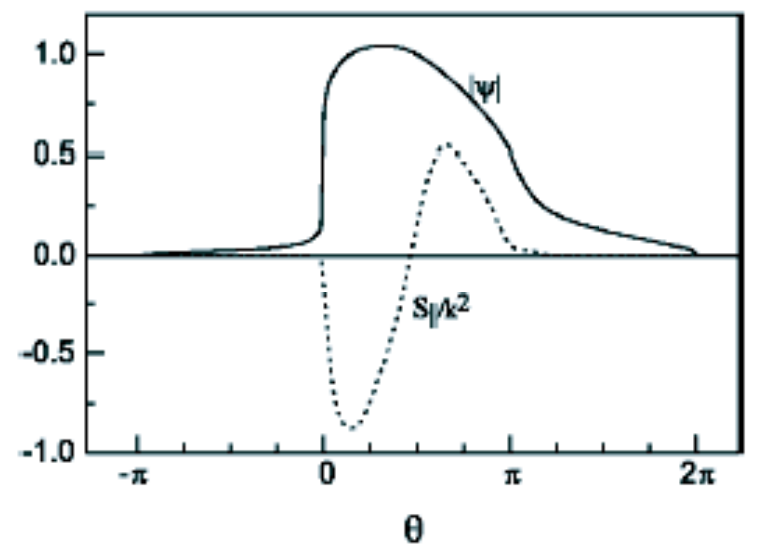

Figure 4. a), The plasma resistivity enhanced by the skin effect, $\eta_{\|} k_{\perp}^{2} c^{2}$, versus poloidal distance for a DIII-D single-null configuration, and b), the corresponding resistive X-point eigenfuction (from Ref [27]). 

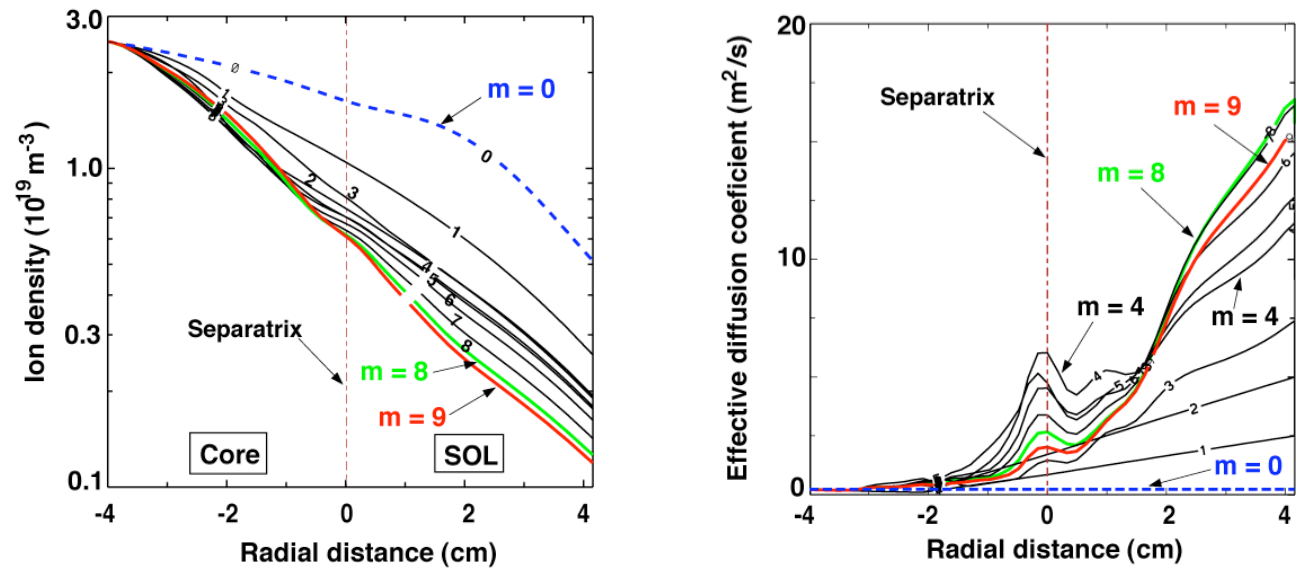

Figure 5. Coupled iterations for 2D edge transport and 3D turbulence showing the steady-state outer midplane density from the transport code and effective particle radial diffusivity from the turbulence code Ref. [33]. The codes are run on their own characteristic timescales with the index $\mathrm{m}$ denoting the iteration number.
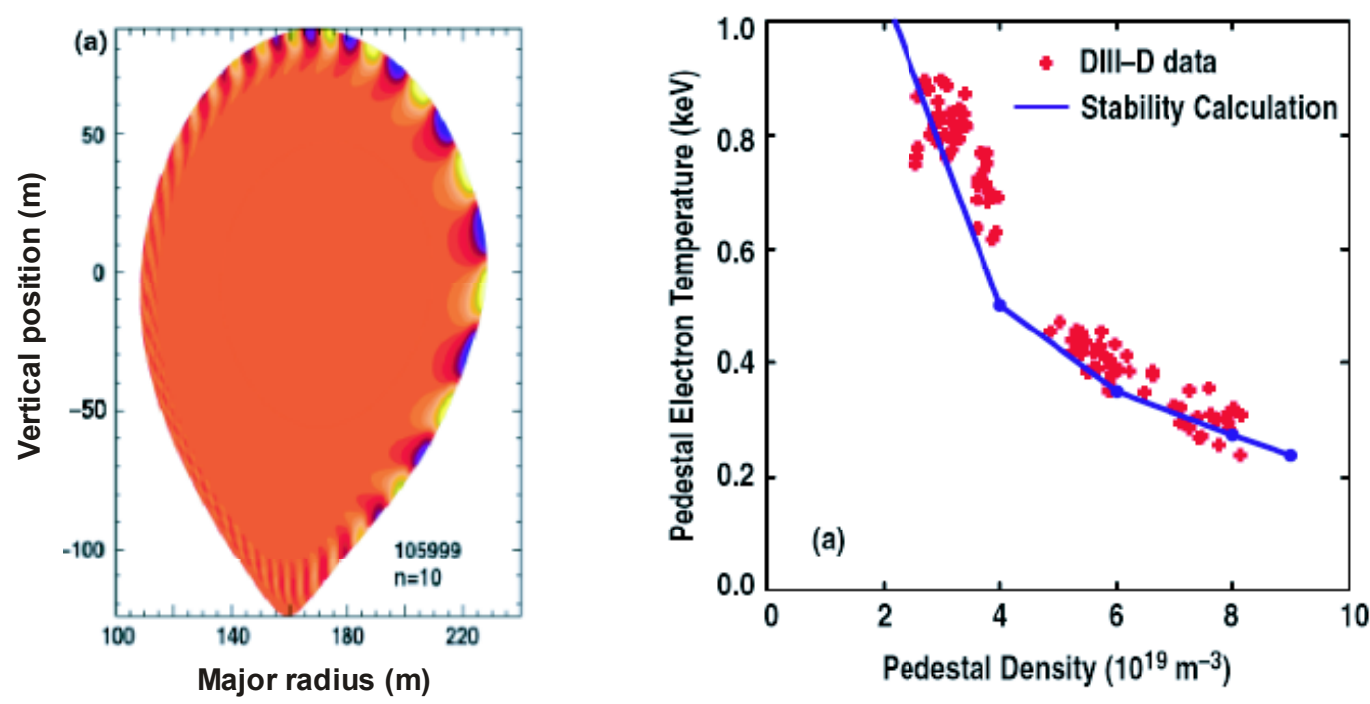

Figure 6. a), plasma resistivity, versus poloidal distance for a DIII-D single-null configuration, and b), the resistive X-point eigenfuction (from Ref. [39]). 